\title{
Investigation of Characteristics of Dual-band Ladder-type Surface Acoustic Wave for High-power Durability Duplexer
}

\author{
Chien-Yu Li, ${ }^{1}$ Hua-Jian Hsu, ${ }^{1}$ Lih-Shan Chen, ${ }^{2}$ and Mau-Phon Houng ${ }^{1 *}$ \\ ${ }^{1}$ Institute of Microelectronics, Department of Electrical Engineering, National Cheng Kung University, \\ No. 1, University Rd., Tainan City 701, Taiwan \\ ${ }^{2}$ Department of Electronic Engineering, I-Shou University, \\ No. 1, Sec. 1, Syuecheng Rd., Dashu District, Kaohsiung City 84001, Taiwan
}

(Received May 30, 2018; accepted October 11, 2018)

Keywords: ADS simulation, ladder type SAW, duplexer, isolation

A circuit investigation of dual-band surface acoustic wave (SAW) filters of the ladder type is proposed. The designed filter exhibits a broader bandwidth and a sharper cut-off frequency than the conventional filter composed of the ladder and double-mode SAW (DMS) types. The SAW filters have been widely applied to the antenna duplexer given the progress in mobile communication technology. Furthermore, the SAW filters can be constructed into a wireless sensor system in combination with a SAW sensor. We demonstrate how simulations of laddertype SAW filters based on the $48^{\circ} \mathrm{YX}-\mathrm{LiTaO}_{3}$ are used to achieve a convenient design of the SAW band-pass filter for a high-power and wireless sensor system. These filters demonstrate the lowest insertion loss, the largest bandwidth, the optimal power durability, and a thirdorder intercept point compared with the interdigital transducer (IDT) and DMS. A simulation of a SAW resonator using the advanced design system (ADS) is performed with the lumped elements. The first passband that is applied to the Tx duplexer is in the range of $823-855 \mathrm{MHz}$, and the second passband applied to the Rx duplexer is from 867 to $898 \mathrm{MHz}$. The frequency characteristics of the ladder-type duplexer possess minimum insertion losses of $1.14 \mathrm{~dB}$ in the first passband and $0.54 \mathrm{~dB}$ in the second passband, which are similar to those of the DMS duplexer. The isolation performance between Tx and $\mathrm{Rx}$ is $42.22 \mathrm{~dB}$ at $823-898 \mathrm{MHz}$, which is less than that of the DMS duplexer. The design of the appropriate SAW band pass filter will be simplified through a precise simulation. Furthermore, the dual-band structure with a laddertype SAW can be expected to endure higher power and have a better isolation performance than conventional DMS structures.

\section{Introduction}

The use of mobile devices has widely affected society given the progress of technology. The high complexity of the frequency domain has resulted in many filter applications. An antenna duplexer of surface acoustic wave (SAW) filters has attracted considerable research attention considering their high power capability, low insertion loss, and steep cut-off properties. ${ }^{(1,2)}$

*Corresponding author: e-mail: mphoung@eembox.ncku.edu.tw https://doi.org/10.18494/SAM.2019.2092 
The SAW devices are also popularly applied in gas sensors, pressure sensors, and biosensors with the piezoelectric characteristic. ${ }^{(3,4)}$ The SAW devices can be applied as gas sensors with relevant materials deposited on the SAW substrate. The sensing mechanism utilizes mass changes due to gas absorption to transform the propagation characteristics. ${ }^{(5-7)} \mathrm{LiTaO}_{3}$ has also been applied to gas sensor production and humidity sensing. ${ }^{(8,9)}$ With their high wave speed, radio frequency identification (RFID) is another significant application of the SAW devices. The change in the structure of a SAW device provides an individual code for each RFID device. SAW devices developed for RFID have been widely used in barcodes, electric wallets, and electronic charging. ${ }^{(10,11)}$ With the many sensor and RF applications, the SAW filter is expected to be used in wireless sensor systems.

SAW duplexers with Tx passbands of $823-855 \mathrm{MHz}$ and Rx passbands from $867-898$ $\mathrm{MHz}$ in the $3 \mathrm{G}$ and $4 \mathrm{G}$ frequency domains have been well developed. Most of the duplexer structures are composed of ladder-type and double-mode SAW (DMS) filters. ${ }^{(12,13)}$ The laddertype SAW filters are composed of numerous SAW resonators that are in serial and parallel arrangements and integrated on a single chip. They exhibit lower insertion losses and higher power capabilities than the DMS structure filters.

Many theories have characterized the transmission of surface waves. In 1885, Lord Rayleigh proposed the mechanical vibration of a SAW. ${ }^{(14)}$ Therefore, many studies simulated the SAW frequency characteristics. Warren P. Mason presented an equivalent circuit to simulate the electrical and mechanical wave properties. ${ }^{(15,16)}$ The Mason model simulated the coupling between the interdigitated transducer with the mechanical and electrical systems. ${ }^{(17)}$

In this research, the simulation of the SAW filter revealed the combination of the Mason equivalent circuit and RLC models with high-accuracy frequency characteristics. The simulation focused on the RLC model to reveal the electrical characteristics of the interdigital transducer (IDT). Furthermore, we simulated a dual-band SAW filter in identical ladder-type structures for high power durability and wireless sensing application.

\section{Resonator Simulation}

The simulation of the ladder-type SAW filter is based on the material of $48^{\circ} \mathrm{YX}-\mathrm{LiTaO}_{3}$, which is a material with a high wave speed of $3900(\mathrm{~m} / \mathrm{s})$ and an 8.2 electromechanical coupling factor. The simulation of the $48^{\circ} \mathrm{YX}-\mathrm{LiTaO}_{3}$ can increase the performance of the filter and reduce the signal loss with high wave speed and electromechanical coupling factor in comparison with the quartz and other piezoelectric materials. The ladder-type SAW filter is composed of two series of SAW resonators in an L-type network. The resonators with high resonance frequency are primarily arranged serially, whereas the resonators with low resonance frequency are arranged in parallel. The serial parts of the SAW resonators obtain slightly different resonance frequencies, thereby indirectly affecting the passband width. We can determine the filter characteristics through appropriate design and simulation. Figure 1(a) illustrates a single SAW device structure consisting of an IDT and a piezoelectric substrate. The definitions of aperture $W$, IDT width, and IDT pair are clearly marked in Fig. 1(b). The schematic of an L-type network duplexer filter is depicted in Fig. 1(c). 


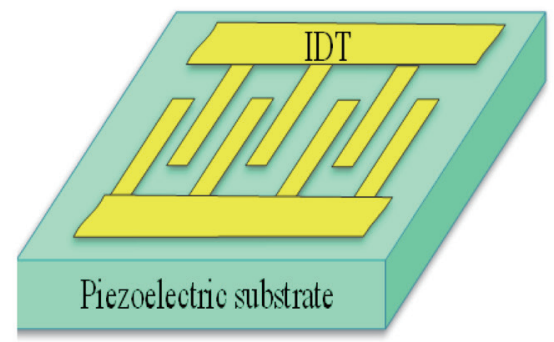

(a)

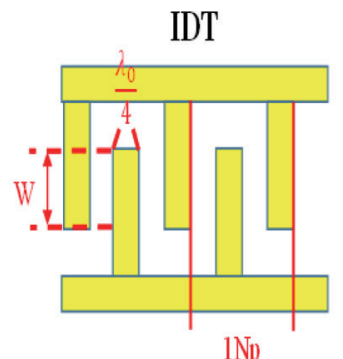

(b)

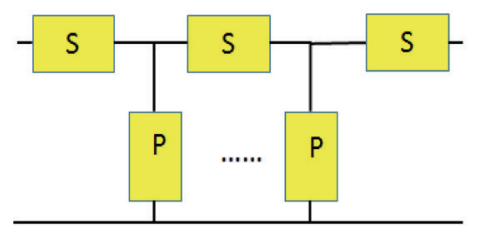

(c)

Fig. 1. (Color online) (a) IDT and piezoelectric substrate structure, (b) pattern parameters of IDT, and (c) schematic of L-type network.

We must begin the simulation with the single SAW resonator to precisely and rapidly design the ladder-type SAW. The single SAW resonator is the most important and basic element in the ladder-type SAW filter. Therefore, we first simulate the SAW resonator using lump devices and their values are calculated using the formulas presented below. ${ }^{(18)}$ These formulas are related to the physical parameters of IDT pairs $N_{p}$, acoustic aperture $W$, wavelength $\lambda_{0}$, electromechanical coupling factor $K^{2}$, wave speed $V$, and capacitance/finger-pair/unit length $C_{o}$. Equation (1) is used to determine the series-resonance frequency of the SAW resonator; this formula is significantly affected by the piezoelectric substrate wave speed $V$ and $\lambda_{0}$. In this research, we set our IDT width and gap to $\lambda_{0} / 4$.

Parallel-resonance frequency $\left(f_{p}\right)$ and series-resonance frequency $\left(f_{s}\right)$ are used to determine filter bandwidth. Thus, we must determine the relationship among $f_{p}, f_{s}$, and physical parameters. Equation (2) indicates that $N_{p}$ will influence the $Q$ factor of the filter, and $d_{e}$ is the effective cavity length (m). The variant of the $Q$ factor and $d_{e}$ significantly affect the width between $f_{p}$ and $f_{s}$ given the modulation of $N_{p}$. The $f_{p}-f_{s}$ bandwidth corresponds to the passband width of the filter.

Figure 2 shows our lump device circuit, and the parameters of devices can be calculated using Eqs. (3)-(6). $C_{T}$ is the static capacitance related to the IDT pair $N_{p}$, capacitance/fingerpair/unit length $C_{o}$, and acoustic aperture $W$.

$L_{r}, C_{r}$, and $R_{r}$ are motional parameters of the resonance circuit. Equation (4) expresses circuit inductance, which is inversely proportional to the electromechanical coupling factor, series-resonance frequency, IDT pairs, $C_{o}$, and acoustic aperture.

$C_{r}$ is the motional capacitance, which causes series resonance with $L_{r} . C r$ and $L_{r}$ are the resonance units and are thus inversely proportional.

Equation (6) indicates that resonator loss is related to the electromechanical coupling factor, acoustic aperture, and IDT pairs. The formula of $R_{r}$ indicates that increasing the acoustic aperture and number of IDT pairs can reduce signal loss. In particular, we can select the substrate with a high electromechanical coupling factor to attain a low loss.

$$
V=f_{0} \times \lambda_{0}(\mathrm{~m} / \mathrm{s})
$$




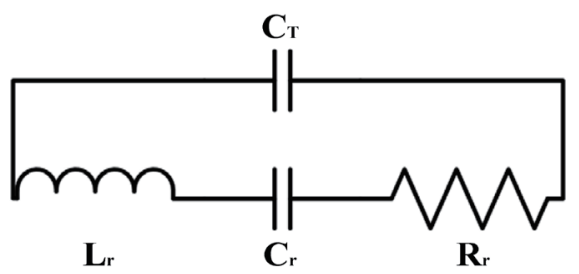

Fig. 2. Lump device model of SAW resonator.

$$
\begin{gathered}
f_{p}-f_{s}=\frac{4 \lambda_{0} K^{2} f_{0} N_{p}^{2}}{d_{e} \pi^{2}}(\mathrm{~Hz}) \\
\mathrm{C}_{T}=N_{p} \times C_{0} \times W(\mathrm{~F}) \\
L_{r}=\frac{d_{e}}{\lambda_{0}} \frac{1}{32 K^{2} f_{0}^{2} N_{p}^{2} C_{o} W}(\mathrm{H}) \\
C_{r}=\frac{1}{4 \pi^{2} f_{0}^{2} L_{r}}(\mathrm{~F}) \\
R_{r}=\frac{1}{8 K^{2} f_{0} C_{o} W N_{p}^{2}}(\Omega)
\end{gathered}
$$

We can quickly simulate the SAW resonator through Eqs. (1)-(6) to calculate the lump device parameters and obtain the physical parameters of the SAW resonator. The actual simulation result of the resonator is illustrated in Fig. 3. This figure depicts the $Y$-parameters of a resonator, in which $f_{s}$ is $836.5 \mathrm{MHz}$ and $f_{p}$ is $864.5 \mathrm{MHz}$.

\section{Ladder-type SAW Simulation}

Equations (1)-(6) can precisely simulate the SAW resonators with the appropriate frequency characteristics. Therefore, we design the ladder-type SAW filter on the basis of the simulated results of the resonators.

In the first step, the center frequency of the filter is decided by the $f_{p}$ of the parallel resonator and the $f_{s}$ of the serial resonator; these resonators are designed individually. The $f_{s}$ of the serial resonator and $f_{p}$ of the parallel resonator must be identical to level the passband. The performance of the filter will be tailored through a special design. The passband width can be modulated with a pair of IDTs. The decrease in $N_{p}$, as exhibited in Fig. 4, is a response to the reduction in $f_{p}-f_{s}$ and the passband width of the filter. We can obtain the proper $f_{p}-f_{s}$ width 


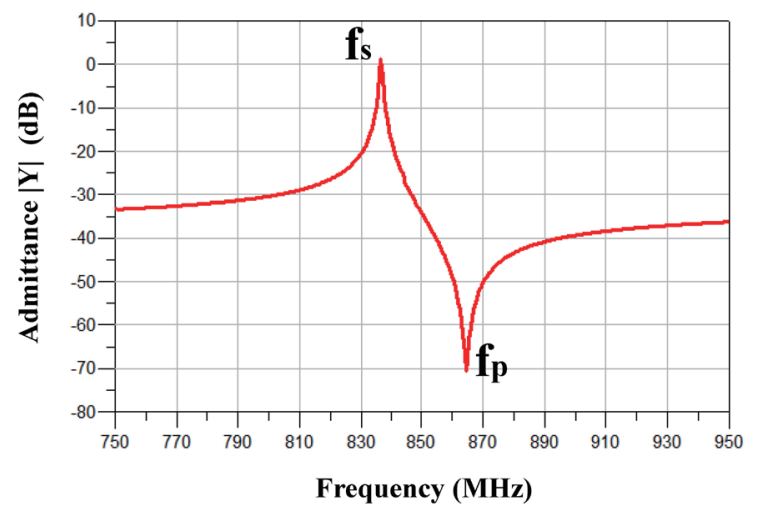

Fig. 3. (Color online) SAW resonator simulation result.

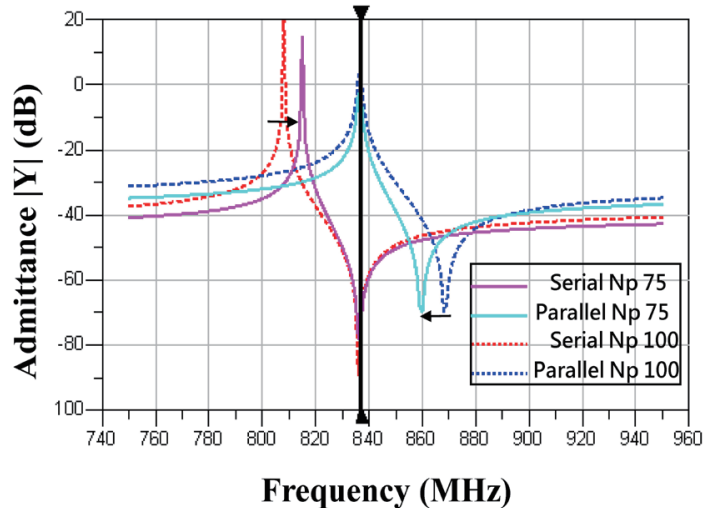

(a)

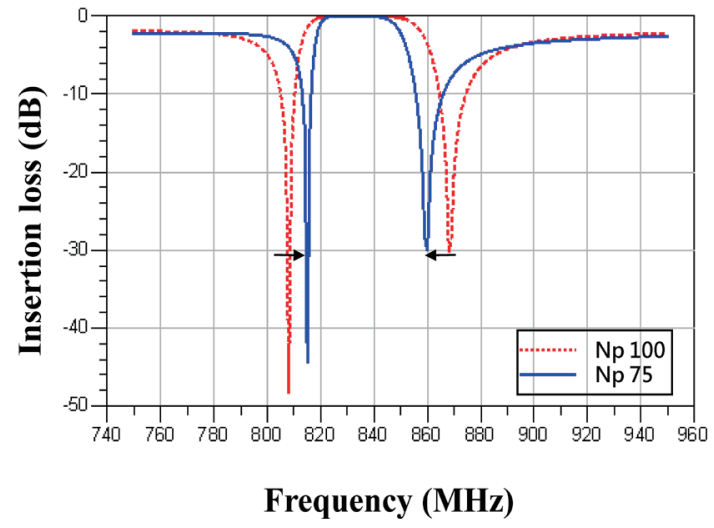

(b)

Fig. 4. (Color online) (a) Resonator admittance and (b) insertion loss of 1-stage ladder-type SAW filter with 75 and 100 pairs of IDTs.

by the fitting of $N_{p}$. We use one band of the duplexer, that is, the Tx ladder-type SAW, whose passband is $823-855 \mathrm{MHz}$. The $f_{s}$ of the serial resonator and $f_{p}$ of the parallel resonator match at $836.5 \mathrm{MHz}$. The $f_{s}$ of the parallel resonator is $814.5 \mathrm{MHz}$ and the $f_{p}$ of the serial resonator is 855.1 MHz, as shown in Fig. 5(a).

We can simply obtain the filter characteristics for our purpose after establishing the method for simulating the resonator and filter. In this research, the duplexer consists of an antenna, a Tx filter, and an Rx filter. We focus on the simulation of the Tx and Rx filters. The Tx and Rx filters are designed using the ladder-type SAW. The Rx filter has a higher frequency band than the Tx filter, and its design flow is the same as that in the previous steps. Figure 5(b) displays the admittance of resonators and insertion loss of the Rx one-stage ladder-type SAW. The $f_{s}$ of the serial resonator overlaps the $f_{p}$ of the parallel resonator.

Figure 5 clearly illustrates that the out-band insertion loss of a one-stage ladder-type SAW is small enough to enable the effective filtering of the signal. We must adjust the circuit for the low insertion loss in the passband and the high loss in the rejection band. The performance of the ladder-type SAW can be optimized by a multiple-stage connection, as shown in Fig. 6. Each 


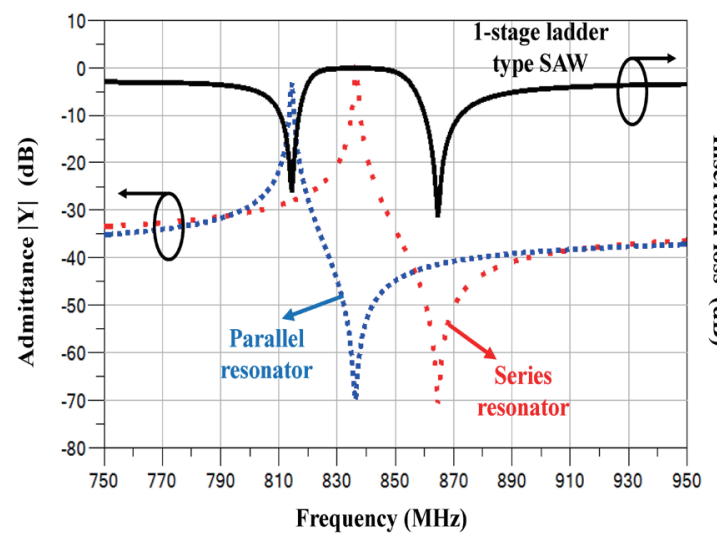

(a)

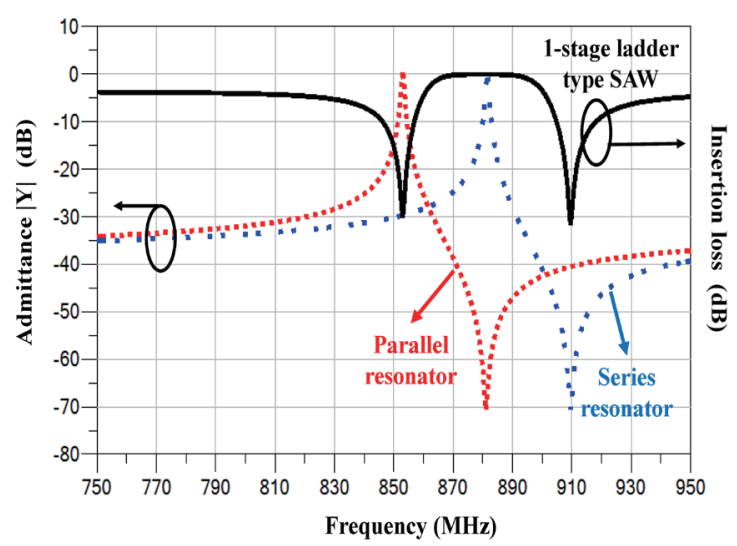

(b)

Fig. 5. (Color online) Resonator admittance and 1-stage ladder-type SAW insertion loss of filter: (a) Tx and (b) Rx.

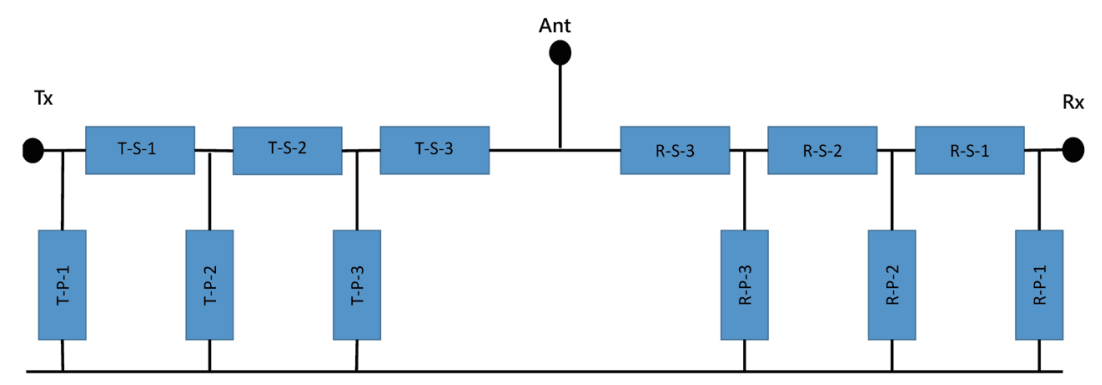

Fig. 6. (Color online) Schematic of four-stage ladder-type duplexer.

Table 1

$N_{p}$ and $W$ of the simulated duplexer.

\begin{tabular}{lcccccc} 
Tx & T-S-1 & T-S-2 & T-S-3 & T-P-1 & T-P-2 & T-P-3 \\
Pair $\left(N_{p}\right)$ & 71 & 70 & 59 & 113 & 143 & 140 \\
Rx & R-S-1 & R-S-2 & R-S-3 & R-P-1 & R-P-2 & R-P-3 \\
Pair $\left(N_{p}\right)$ & 81 & 80 & 65 & 139 & 160 & 117 \\
\hline
\end{tabular}

resonator indicates a variety of $N_{p}$, as listed in Table 1 , which can be obtained after fitting the bandwidth and the insertion loss of the passband. The fitting of $N_{p}$ yields the passband width of the duplexer. We can obtain an extremely low insertion loss in the passband and a relatively high loss in the rejection band after the multiple-stage connection in our circuit. Figure 7 demonstrates the insertion loss of the Rx and Tx antennas in the multiple-stage connection. In the figure, the passband indicates an insertion loss performance extremely close to and a bandwidth identical to the measured results. The Tx passband is in the range of 823-855 MHz, which indicates a minimum insertion loss of approximately $1.14 \mathrm{~dB}$. The Rx passband is in the range of $867-898 \mathrm{MHz}$, denoting a minimum insertion loss of approximately $0.54 \mathrm{~dB}$. The measurement result exhibits a valley in the middle of the passband, caused by the impedance mismatch in the measurement system. The simulation can further alleviate the drawback by increasing the acoustic aperture $W$. With an increasing aperture in each resonator, the $R_{r}$ 


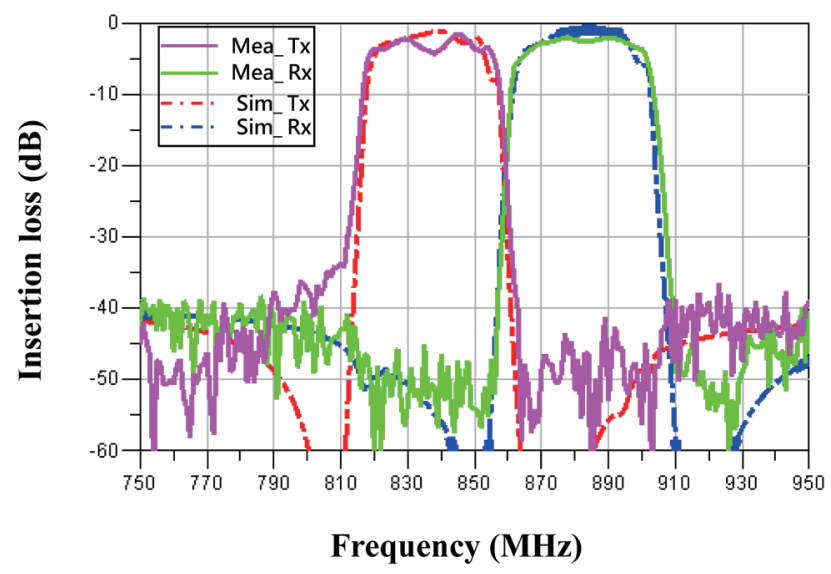

Fig. 7. (Color online) Multiple-stage ladder-type SAW filter characteristic with Tx and Rx antennas.

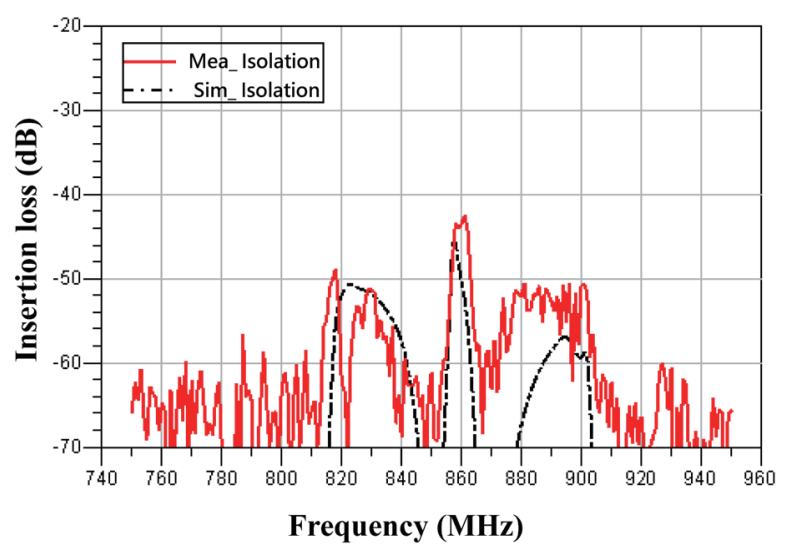

Fig. 8. (Color online) Isolation of Tx and Rx.

Table 2

Frequency responses of simulated ladder-type, measured ladder-type, and DMS duplexers.

\begin{tabular}{lcccc}
\hline Duplexer form & Insertion loss $(\mathrm{dB})$ & Isolation $(\mathrm{dB})$ & Power durability & Attenuation $(\mathrm{dB})$ \\
\hline Sim_ladder type & $0-2$ & $<45$ & $>1 \mathrm{~W}$ & $\sim 40$ \\
Mea_ladder type & $1-3$ & $<42$ & $>1 \mathrm{~W}$ & $\sim 40$ \\
DMS & $2-3$ & $<20$ & $<50 \mathrm{~mW}$ & $\sim 50$ \\
\hline
\end{tabular}

can be fitted into the most suitable value and the valley in the middle of the passband can be eliminated.

The isolation of $\mathrm{Tx}$ and $\mathrm{Rx}$ is a crucial property of the duplexer. The ladder-type filters have lower insertion losses than the DMS-type filters at the outband frequency. Therefore, the isolation of the ladder-type duplexer is expected to be better than that of the DMS-type duplexer. Figure 8 shows the simulated and measured isolation between Tx and Rx. The insertion loss in the simulation is less than $42.22 \mathrm{~dB}$. This insertion loss of isolation is lower than the measured result. To improve the isolation, we add a transmission line between the antenna and Rx laddertype SAW to achieve impedance matching. ${ }^{(19)}$ The characteristics of isolation and the Tx filter can be significantly improved by adding the transmission line. In Table 2, we summarize the performance of the ladder-type duplexer, both simulated and measured, in comparison with that of the DMS-type duplexer. The ladder-type duplexer can achieve a low loss at the passbands of 823-855 and 867-898 MHz, and a $40 \mathrm{~dB}$ attenuation of the outband was also achieved in both simulation and measurement, similarly to the DMS type. The isolation of the ladder type is less than $40 \mathrm{~dB}$, which is a much better performance than the $20 \mathrm{~dB}$ of the DMS duplexer.

\section{Conclusions}

In this research, we proposed a rapid simulation method for a duplexer system fabricated with a ladder-type SAW filter applied to the Tx and Rx passbands. The ladder-type structure exhibits excellent performance, that is, a lower insertion loss at the passband and better isolation 
between Tx and Rx than that of the DMS structure. The simulation utilizes the electronic characteristics of the SAW device are demonstrated by advanced design system (ADS) software. The ladder-type SAW is simulated using a resonator that is composed of passive RLC devices. The passband of the filter can be controlled via the appropriate arrangement of the SAW resonator in an L-type network. The frequency characteristic can be further improved by arranging multiple stages of the L-type network into a ladder-type form. By selecting a suitable $N_{p}$ of each resonator in the SAW filter, we can significantly adjust the bandwidth and insertion loss of the passband. The results show that the Tx and Rx antennas indicate wide bandwidths of $823-855 \mathrm{MHz}$ in the first passband and $867-898 \mathrm{MHz}$ in the second passband. The low insertion losses in the first and second passbands are 1.14 and $0.54 \mathrm{~dB}$, respectively. The isolation between Tx and Rx is less than $42.22 \mathrm{~dB}$. The appropriate specifications of the laddertype SAW filter can be rapidly simulated and precisely match the measurement results. The measurement results of the ladder-type duplexer also indicate its better frequency response than that of the DMS structure duplexer.

\section{References}

1 J. Tsutsumi, S. Inoue, Y. Iwamoto, T. Matsuda, M. Miura, Y. Satoh, M. Ueda, and O. Ikata: IEEE Int. Frequency Control Symp. and PDA Exhibition Jointly with the 17th European Frequency and Time Forum (IEEE, 2003) 861-867. https://doi.org/10.1109/FREQ.2003.1275203

2 T. Matsuda, J. Tsutsumi, S. Inoue, Y. Iwamoto, Y. Satoh, M. Ueda, and O. Ikata: IEEE Proc. Ultrason. Symp. (IEEE, 2002) 68-73. https://doi.org/10.1109/ULTSYM.2002.1193355

3 W. Wang, K. Lee, S. Yang, and I. Park: Sens. Mater. 18 (2006) 301. https://myukk.org/SM2017/sm_pdf/SM651. pdf

4 T. Han, X. Ji, and W. Shi: Sens. Mater. 18 (2006) 173. https://pdfs.semanticscholar.org/ b9c3/891f2d89ce28e1ae7f54b75ad4cb0278e97d.pdf

5 R. Arsat, M. Breedon, M. Shafiei, P. G. Spizziri, S. Gilje, R. B. Kaner, K. Kalantar-zadeh, and W. Wlodarski: Chem. Phys. Lett. 467 (2009) 344. https://doi.org/10.1016/j.cplett.2008.11.039

6 A. Z. Sadek, W. Wlodarski, Y. X. Li, W. Yu, X. Li, X. Yu, and K. Kalantar-zadeh: Thin Solid Films 515 (2007) 8705. https://doi.org/10.1016/j.tsf.2007.04.009

7 W. Wen, H. Shitang, L. Shunzhou, L. Minghua, and P. Yong: Sens. Actuators, B 125 (2007) 422. https://doi. org/10.1016/j.snb.2007.02.037

8 Y. Wang, S.-Y. Zhang, F.-M. Zhou, L. Fan, Y.-T. Yang, and C. Wang: Sens. Actuators, B 158 (2011) 97. https:// doi.org/10.1016/j.snb.2011.05.047

9 S. J. Ippolito, A. Ponzoni, K. Kalantar-Zadeh, W. Wlodarski, E. Comini, G. Faglia, and G. Sberveglieri: Sens. Actuators, B 117 (2006) 442. https://doi.org/10.1016/j.snb.2005.12.050

10 L. Reindl, G. Scholl, T. Ostertag, H. Scherr, U. Wolff, and F. Schmidt: IEEE Trans. Ultrason. Ferroelectr. Freq. Control 45 (1998) 1281. https://doi.org/10.1109/58.726455

11 A. Pohl: IEEE Trans. Ultrason. Ferroelectr. Freq. Control 47 (2000) 317. https://doi.org/10.1109/58.827416

12 S. Inoue, J. Tsutsumi, Y. Iwamoto, T. Matsuda, M. Miura, Y. Satoh, M. Ueda, and O. Ikata: 2003 IEEE Symp. Ultrasonics (2003) 389-392. https://doi.org/10.1109/ULTSYM.2003.1293429

13 N. Shibagaki, T. Akagi, K. Hasegawa, K. Sakiyama, and M. Hikita: IEEE Proc. Ultrason. Symp. 1 (1994) 129-134. https://doi.org/10.1109/ULTSYM.1994.401565

14 Lord Rayleigh: Proc. London Math. Soc. 1 (1885) 4. https://doi.org/10.1112/plms/s1-17.1.4

15 W. P. Mason: Electromechanical Transducers and Wave Filters (Van Nostrand, New York, 1948).

16 W. P. Mason: J. Acoust. Soc. Am. 28 (1956) 1197. https://doi.org/10.1121/1.1908593

17 L. W. Marshall: IEEE Trans. Ultrason. Ferroelectr. Freq. Control 41 (1994) 60. https://doi. org $/ 10.1109 / 58.265821$

18 E. J. Staples, J. S. Schoerwald, R. C. Rosenfeld, and C. S. Hartmann: IEEE Proc. Ultrason. Symp. (1974) 245252. https://doi.org/10.1109/ULTSYM.1974.196329

19 S. Inoue, J. Tsutsumi, T. Matsuda, M. Ueda, O. Ikata, and Y. Satoh: IEEE Trans. Ultrason. Ferroelectr. Freq. Control 54 (2007) 9. https://doi.org/10.1109/TUFFC.2007.472 